\title{
INFORMATION THEORETIC MEAN SHIFT ALGORITHM
}

\author{
Sudhir Rao, Weifeng Liu, Jose C. Principe \\ CNEL, Department of ECE \\ University of Florida \\ Gainesville, Florida, U.S.A
}

\author{
Allan de Medeiros Martins* \\ Department of ECE \\ Federal University of Rio Grande do Norte \\ Natal, RN, Brazil
}

\begin{abstract}
In this paper we introduce a new cost function called Information Theoretic Mean Shift algorithm to capture the "predominant structure" in the data. We formulate this problem with a cost function which minimizes the entropy of the data subject to the constraint that the Cauchy-Schwartz distance between the new and the original dataset is fixed to some constant value. We show that Gaussian Mean Shift and the Gaussian Blurring Mean Shift are special cases of this generalized algorithm giving a whole new perspective to the idea of mean shift. Further this algorithm can also be used to capture the principal curve of the data making it ubiquitous for manifold learning.
\end{abstract}

\section{INTRODUCTION}

Consider a dataset $X_{o}=\left(x_{i}\right)_{i=1}^{N} \in \mathbf{R}^{D}$. Using the nonparametric method of parzen window technique, the probability density estimate is given by

$$
p(x)=\frac{1}{N} \sum_{i=1}^{N} G\left(\left\|\frac{x-x_{i}}{\sigma}\right\|^{2}\right)
$$

where $G(t)=e^{-\frac{t}{2}}$ is a gaussian kernel with bandwidth $\sigma>0$. In order to find the modes of the pdf we rearrange the stationary point equation $\nabla p(x)=0$ into an iterative fixed point scheme $x^{(\tau+1)}=m\left(x^{(\tau)}\right)$ with

$$
m(x)=\frac{\sum_{i=1}^{N} G\left(\left\|\frac{x-x_{i}}{\sigma}\right\|^{2}\right) x_{i}}{\sum_{i=1}^{N} G\left(\left\|\frac{x-x_{i}}{\sigma}\right\|^{2}\right)}
$$

The difference $m(x)-x$ is called mean shift in Fukunaga and Hostetler [1].In his 1975 landmark paper, Fukunaga showed that this recursive algorithm is a steepest ascent technique moving the data points closer to their respective modes at each iteration. Following the discussions in [2], this algorithm is called the Gaussian Blurring Mean Shift algorithm (GBMS) indicating the successive blurring of the dataset towards its respective modes.

Fukunaga's algorithm was largely forgetten, when in 1995, Cheng et al.[2] rekindled the interest by introducing

\footnotetext{
* The author performed the work while at CNEL, University of Florida.
}

a slight change in the way the iterative scheme was calculated. Instead of using the changed dataset at every iteration to calculate the mean shift of the data points as done in GBMS, Cheng used the original dataset $X_{o}$. This algorithm was called Gaussian Mean Shift (GMS).

Thus in GBMS, the algorithm is initialized to $X^{(0)}=$ $X_{o}$ after which the original dataset is forgotten. As the algorithm is allowed to run we get the blurred datasets $X^{(1)}, X^{(2)}$ and so on. Whereas in GMS, two different datasets are maintained namely $Y$ and $X_{o}$. The dataset $\mathrm{Y}$ is initialized to $X_{o}$ i.e $Y^{(0)}=X_{o}$. As the algorithm is allowed to run, at every iteration $Y^{(\tau)}$ is compared to $X_{o}$ to produce $Y^{(\tau+1)}$.

Following these two papers, a spurt of activities commenced in this field leading to many interesting applications. Carreira-Perpiñán et al. used mean shift for mode finding in mixture of Gaussian distributions [3]. Their connection to Nadarayana-Watson estimator from kernel regression and the robust M-estimators of location has been thoroughly explored by Comaniciu et al. [4].

Mean Shift algorithms has been successfully applied in image segmentation and denoising [4], real-time tracking of non-rigid objects [5] and other computer vision applications [6]. Its simplicity, ability to deal with clusters of arbitrary shape and a single parameter (kernel size) to control the scale of analysis makes it particularly attractive and suitable for wide range of applications.

Although these algorithms are simple and successful, they lack the intuitive reasoning as to what do they optimize. For example, its not clear what change is incurred when going from GMS to GBMS and vice versa. In [2] and [4], the authors try to address this issue with various postulates and optimization concepts making the analysis very complex. Thus, in this regard, the goal of our paper is two folded. Firstly, through our new cost function we develop a broader class of algorithms of which GMS and GBMS are special cases and secondly, we bring a whole new perspective to these algorithms making their reasoning more intuitive and appealing.

The next section introduces Renyi's quadratic entropy and information potential concepts. Section 3 introduces our new cost function and derives its fixed point update rule. We further analyze some special cases of this algorithm. We examine various results in section 4 and finally conclude in section 5 . 


\section{RENYI'S QUADRATIC ENTROPY AND INFORMATION POTENTIAL}

Renyi's quadratic entropy for a pdf is defined as [7]

$$
H(X)=-\log \left(\int p^{2}(x) d x\right)
$$

where the pdf estimate is given as in (1). Using this result and the fact that the integral of the product of two Gaussians is another Gaussian with a variance equal to the sum of the variances, yields a non parametric estimator for Renyi's quadratic entropy

$$
\begin{aligned}
H(X) & =-\log (V(X)) \\
V(X) & =\frac{1}{N^{2}} \sum_{i=1}^{N} \sum_{j=1}^{N} G\left(\left\|\frac{x_{i}-x_{j}}{\sigma^{\prime}}\right\|^{2}\right)
\end{aligned}
$$

where $\sigma^{\prime}=\sqrt{2} \sigma$. Principe et al. and collaborators [8][9] named $V(X)$ as the information potential of the pdf $p(x)$, an analogy borrowed from physics for potential of group of interacting particles. Note that the potential experienced by a single data point due to all other particles is given by

$$
V\left(x_{i}\right)=\frac{1}{N^{2}} \sum_{j=1}^{N} G\left(\left\|\frac{x_{i}-x_{j}}{\sigma^{\prime}}\right\|^{2}\right)
$$

The derivative of this contribution with respect to the value of this sample is easily evaluated to be

$$
\begin{aligned}
\frac{\partial}{\partial x_{i}} V\left(x_{i}\right) & =\frac{1}{N^{2}} \sum_{j=1}^{N} G\left(\left\|\frac{x_{i}-x_{j}}{\sigma^{\prime}}\right\|^{2}\right)\left(\frac{x_{j}-x_{i}}{\sigma^{\prime}}\right) \\
F\left(x_{i}\right) & =\sum_{j=1}^{N} F\left(x_{i} \mid x_{j}\right)
\end{aligned}
$$

where $F\left(x_{i} \mid x_{j}\right)$ is the "information force" exerted by particle $x_{j}$ on particle $x_{i}$ and $F\left(x_{i}\right)$ is the net force experienced by particle $x_{i}$.

Following similar arguments, one can derive the equations for "cross" entropy which are given below.

$$
\begin{aligned}
H\left(X ; X_{o}\right) & =-\log \left(V\left(X ; X_{o}\right)\right) \\
V\left(X ; X_{o}\right) & =E_{p(X)}[q(X)]=\int p(x) q(x) d x \\
& =\frac{1}{N^{2}} \sum_{i=1}^{N} \sum_{j=1}^{N} G\left(\left\|\frac{x_{i}-x_{o j}}{\sigma^{\prime}}\right\|^{2}\right)
\end{aligned}
$$

where $p(x)$ and $q(x)$ are the pdfs of $X$ and $X_{o}$ respectively. Thus the information potential and force experienced by individual particle is given by

$$
\begin{aligned}
& V\left(x_{i} ; X_{o}\right)=\frac{1}{N^{2}} \sum_{j=1}^{N} G\left(\left\|\frac{x_{i}-x_{o j}}{\sigma^{\prime}}\right\|^{2}\right) \\
& F\left(x_{i} ; X_{o}\right)=\sum_{j=1}^{N} F\left(x_{i} \mid x_{o j}\right)
\end{aligned}
$$

Before we complete this section, we define the CauchySchwartz distance measure [8] between two pdfs as

$$
\begin{aligned}
D_{c s}\left(X, X_{o}\right) & =\log \left(\frac{\left(\int p^{2}(x) d x\right)\left(\int q^{2}(x) d x\right)}{\left(\int p(x) q(x) d x\right)^{2}}\right) \\
& =-\left[H(X)+H\left(X_{o}\right)-2 H\left(X ; X_{o}\right)\right]
\end{aligned}
$$

\section{INFORMATION THEORETIC MEAN SHIFT ALGORITHM (ITMS)}

Consider an original dataset $X_{o}=\left(x_{o j}\right)_{j=1}^{N} \in \mathbf{R}^{D}$. Our aim is to find a dataset $X=\left(x_{i}\right)_{i=1}^{M} \in \mathbf{R}^{D}, M \leq N$ that captures the "structure" in $X_{o}$. The immediate question which arises is how much of the "structure" we wish to capture? At one extreme, we could represent the cluster with only a single particle i.e $M=1$ and on the other extreme we could represent it with the dataset itself i.e $M=N$. We formulate this idea as minimizing the entropy of the dataset $X$ with the constraint that the Cauchy-Schwartz distance between $X$ and $X_{o}$ is held to some constant value $\mathrm{k}$ with $0 \leq k \leq H\left(X_{o}\right)$ i.e

$$
\min _{X} H(X) \text { subject to } D_{c s}\left(X, X_{o}\right)=k
$$

This constrained optimization problem can be formulated as an unconstrained optimization using Lagrange multiplier as shown below.

$$
\begin{aligned}
J(X)= & \min _{X} H(X)+\lambda\left[D_{c s}\left(X, X_{o}\right)-k\right] \\
= & \min _{X} H(X)-\lambda\left[\left(H(X)+H\left(X_{o}\right)-2 H\left(X ; X_{o}\right)\right]\right. \\
& \quad-\lambda k \\
= & \min _{X}(1-\lambda) H(X)+2 \lambda H\left(X ; X_{o}\right)-\lambda H\left(X_{o}\right)-\lambda k \\
= & \min _{X}-(1-\lambda) \log (V(X))-2 \lambda \log \left(V\left(X ; X_{o}\right)\right) \\
& \quad+\lambda \log \left(V\left(X_{o}\right)\right)-\lambda k
\end{aligned}
$$

Differentiating $J(X)$ with respect to each $x_{i}$ gives a simple fixed point update rule.

$$
\frac{\partial}{\partial x_{i}} J(X)=-\frac{(1-\lambda)}{V(X)} F\left(x_{i}\right)-\frac{2 \lambda}{V\left(X ; X_{o}\right)} F\left(x_{i} ; X o\right)
$$




$$
x_{i}^{\tau+1}=\frac{c_{1} \sum_{j=1}^{N} G\left(\left\|\frac{x_{i}^{\tau}-x_{j}^{\tau}}{\sigma^{\prime}}\right\|^{2}\right) x_{j}^{\tau}+c_{2} \sum_{j=1}^{N} G\left(\left\|\frac{x_{i}^{\tau}-x_{o j}}{\sigma^{\prime}}\right\|^{2}\right) x_{o j}}{c_{1} \sum_{j=1}^{N} G\left(\left\|\frac{x_{i}^{\tau}-x_{j}^{\tau}}{\sigma^{\prime}}\right\|^{2}\right)+c_{2} \sum_{j=1}^{N} G\left(\| \frac{\left.\left\|\frac{x_{i}^{\tau}-x_{o j}}{\sigma^{\prime}}\right\|^{2}\right)}{(13)}\right.}
$$

where $c_{1}=\frac{(1-\lambda)}{V(X)}$ and $c_{2}=\frac{2 \lambda}{V\left(X ; X_{o}\right)}$. We start the algorithm by initializing $X=X_{o}$. Before we consider the general case, lets look at some special cases of interest.

\subsection{When $\lambda=0$}

When $\lambda=0$ the cost function simplifies to

$$
J(X)=\min _{X} H(X)
$$

For a Gaussian kernel with infinite support, this leads to a single point as the global solution since then $H(X)=0$. Using the fact that log is monotonous function, minimization of $H(X)$ is equal to maximization of $V(X)$. Thus the cost function becomes

$$
J(X)=\max _{X} V(X)
$$

Taking the derivative with respect to the each data point $x_{i}$ and equating to zero gives the following fixed point update

$$
\begin{gathered}
\frac{\partial}{\partial x_{i}} J(X)=F\left(x_{i}\right)=0 \\
x_{i}^{\tau+1}=\frac{\sum_{j=1}^{N} G\left(\left\|\frac{x_{i}^{\tau}-x_{j}^{\tau}}{\sigma^{\prime}}\right\|^{2}\right) x_{j}^{\tau}}{\sum_{j=1}^{N} G\left(\left\|\frac{x_{i}^{\tau}-x_{j}^{\tau}}{\sigma^{\prime}}\right\|^{2}\right)}
\end{gathered}
$$

This expression could also be obtained by directly plugging $\lambda=0$ in (13). Comparing the above fixed point update with $m(x)$ in (2), we see that this is nothing but GBMS algorithm. Thus the GBMS algorithm tries to minimize the overall entropy of the data. Since the only global solution to this optimization is a single point, we conclude that GBMS is an unstable algorithm and care should be taken to stop the algorithm at the appropriate iteration if the interest is to find the modes of the data.

\subsection{When $\lambda=1$}

When $\lambda=1$ the cost function simplifies to

$$
J(X)=\min _{X} H\left(X ; X_{o}\right)
$$

A similar analysis would lead us to the following fixed point update which can be obtained directly by substituting this value of $\lambda$ in (13).

$$
x_{i}^{\tau+1}=\frac{\sum_{j=1}^{N} G\left(\left\|\frac{x_{i}^{\tau}-x_{o j}}{\sigma^{\prime}}\right\|^{2}\right) x_{o j}}{\sum_{j=1}^{N} G\left(\left\|\frac{x_{i}^{\tau}-x_{o j}}{\sigma^{\prime}}\right\|^{2}\right)}
$$

This is in fact the GMS algorithm where the point of the current dataset is compared to the original dataset to compute its mean shift. In GMS algorithm, the shape of the dataset is remembered at each iteration as embodied in the "cross" entropy $H(X ; X o)$. At every iteration, the mean shift vector gives direction to the particle with respect to the original pdf. Thus one of the stable solutions of this algorithm are the modes of the pdf. No special stopping criterion is required except to stop the algorithm when it is running unnecessarily which can be easily done when the change in position of the particle are below some tol level.

\subsection{When $\lambda>1$}

When $\lambda>1$, an interesting scenario develops. Consider again the cost function

$$
\begin{aligned}
J(X) & =\min _{X} H(X)+\lambda\left[D_{c s}\left(X, X_{o}\right)-k\right] \\
& =\min _{X}(1-\lambda) H(X)+2 \lambda H\left(X ; X_{o}\right)-\lambda H\left(X_{o}\right)-\lambda k
\end{aligned}
$$

As the value of $\lambda$ increases, more and more weight is being given to the constraint of similarity between $X$ and $X_{o}$ i.e $D_{c s}\left(X, X_{o}\right)$. This is accomplished by representing more and more relevant features of the dataset $X_{o}$. As $\lambda$ increases from 1 to 2 the modes give way to the principal curve of the data. Further increasing the $\lambda$ value makes the data represent more finely the denser regions in the pdf. In short, every fine nuances of the pdf is captured as the $\lambda$ value is increased.

Another interesting way of looking at this scenario is through the interaction of forces. As seen in the second line of (20) when $\lambda>1$ the first term $H(X)$ is negative whereas the second term is $H\left(X ; X_{o}\right)$ is positive. Thus we are maximizing the entropy and minimizing the "cross" entropy at the same time. These opposing forces would make the dataset $X$ settle in some stationary solution.

\section{EXPERIMENTAL RESULTS}

To illustrate our concepts we have selected a dataset having two crescent shaped clusters as shown in Fig. 1(a). We fix our scale of analysis by selecting $\sigma^{2}=0.05$. Fig. $1(b)$ shows the pdf estimation with this particular value of sigma. We would now like to seek the modes of this pdf using GMS and GBMS methods.

\subsection{GMS}

Recall that GMS is a stable mode seeking algorithm and corresponds to the case of $\lambda=1$ in our cost function. The stopping criterion is to stop when the averaged norm distance for the particle is less than some tol level. Equation below clarifies this idea. 


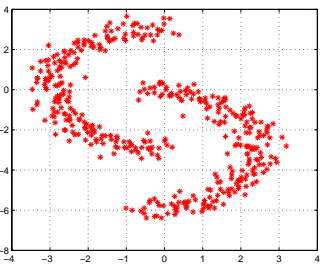

(a) Crescent shaped Dataset

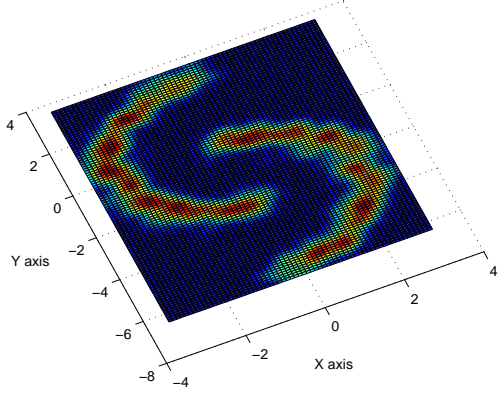

(b) pdf estimation using $\sigma^{2}=0.05$

Fig. 1. Crescent dataset and its pdf estimation

$$
\begin{gathered}
\text { Stop when } \frac{1}{N} \sum_{i=1}^{N} d^{(\tau)}\left(x_{i}\right)<\text { tol where } \\
d^{(\tau)}\left(x_{i}\right)=\left\|x_{i}^{(\tau)}-x_{i}^{(\tau-1)}\right\|
\end{gathered}
$$

We set the $t o l=10^{-6}$ and after 98 iterations the algorithms stopped with the result shown in Fig. 2. Comparing this to Fig. 1(b) we see that the algorithm has found all the modes precisely. The algorithm gives the same solution no matter how many more iterations we run since these are the stationary points of the pdf and hence the global solution of our new cost function.

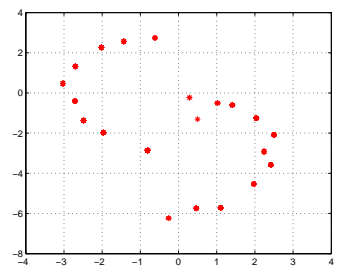

Fig. 2. Mode Seeking ability of GMS algorithm

\subsection{GBMS}

As seen previously, GBMS corresponds to $\lambda=0$ in our cost function. We use the same stopping criteria as given in (21).
Since there is no stable solution of GBMS except a single point, setting the tol level is tricky. After some trials, we found that a tol level of $10^{-3}$ gives the best mode seeking ability of GBMS as shown in Fig. 3a. The number of iterations was 14 which shows the high speed of convergence of GBMS compared to GMS.

On the negative aspect there are two important things to notice. Firstly, the number of modes identified by GBMS is lesser than GMS. For example GBMS combined two important modes located at $0<X<1.5$ and $Y=-6$ approximately into a single mode. Secondly, the positions of the modes do not precisely match up with the modes of the original pdf.The reason for this is that apart from the particles moving towards the modes, the modes themselves are slowly moving towards each other thus making GBMS very unstable.

From the above discussion we conclude that GBMS improves the speed by sacrificing stability compared to GMS algorithm. To substantiate this claim, we ran the GBMS algorithm for 100 iterations instead of stopping it at $14^{\text {th }}$ iteration. The result is shown in Fig. $3 b$. Not only several important modes got combined but also their positions have been altered. Further, selecting the tol level is very heuristic in GBMS and changes with data making "true" mode finding extremely difficult.

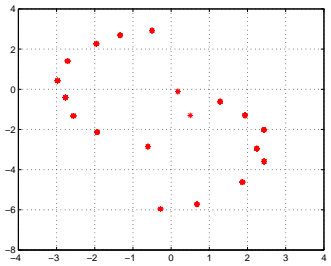

(a)Poor Mode Seeking ability of GBMS algorithm

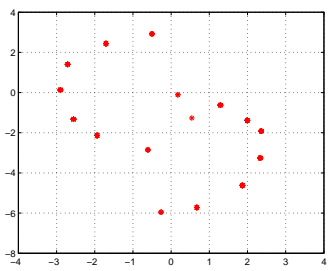

(b) Instability of GBMS algorithm

Fig. 3. Performance of the GBMS algorithm

\subsection{Principal Curves}

A very interesting offshoot of our algorithm is the ability to find principal curve of the data. Principal curves were first defined by Hastie and Stuetzle [10] as "self-consistent" smooth curves which pass through the "middle" of a ddimensional probability distribution or data cloud. HastieStuetzle Algorithm attempts to minimize the average squared 
distance of the data points and the curve. It also uses two different smoothing techniques to avoid overfitting.

Our motivation comes from the fact that every data has the information of its principal curve. Thus in our cost function, by decreasing the entropy of the data and at the same time giving more weightage to the similarity measure part, we can in essence capture the principal curve of the data. To do this we use $1<\lambda<3$. To satisfy the smoothness constraint we use $\sigma^{2}=0.5$, thus making the pdf estimation smooth.

Fig. 4 shows the result on our crescent dataset obtained with $\lambda=2$. The algorithm ran for 40 iterations when the tol level was set at $10^{-3}$. Clearly, the data points now trace out a smooth trajectory of the principal curve which can be easily fit using a simple curve fitting algorithm.

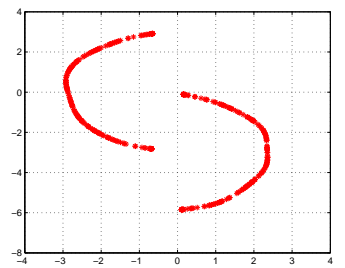

Fig. 4. Principal curve of crescent dataset

\subsection{Effect of increasing $\lambda$}

We next consider the effect of increasing $\lambda$. As described earlier, increasing $\lambda$ gives more weightage to the CauchyScwartz distance constraint making the data points represent more and more relevant features of the dataset. We ran the simulation for 30 different values of $\lambda$ between 2 and 20 .

Here we need to emphasize an important issue. We found that our fixed point update rule for large values of $\lambda$ made our data points to oscillate drastically making it very unstable. Its not very clear whether this is the effect of perturbation error in fixed point algorithm or due to our implementation. In [1], the author stresses that same result is obtained as fixed point update rule if one used a gradient descent technique with small step size, though it may take many iterations to converge as was observed practically. Thus we use the following gradient based rule.

$$
x_{i}^{(\tau+1)}=x_{i}^{(\tau)}-\mu \frac{\partial}{\partial x_{i}} J(X)
$$

where the step size $\mu$ was selected to be 0.1 . Fig. 5 shows the result for 6 different values of $\lambda$. In each case the algorithm was run for 1000 iterations since there was no perceptible change observed for more iterations. Fig. 5(a) shows a better fitting principal curve for the crescent data. As the $\lambda$ was further increased, the denser regions were finely represented as seen in Fig. 5(b) where the principal curve starts splitting at the dense region of second cluster.
As this process continues, the data points attempt to capture every fine nuances of the pdf, in turn representing the pdf extremely well.

\subsection{Denoising property}

Fig. 6(a) shows the "chain of rings" dataset in 3 dimensions with gaussian noise of variance 1 added to it. We use a $\lambda=1.5$ and $\sigma^{2}=1$. Our algorithm successfully removes noise and extracts the underlying "principal manifold" as seen in Fig. 6(b).

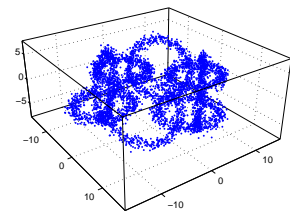

(a) Noisy $3 D$ dataset "chain of rings"

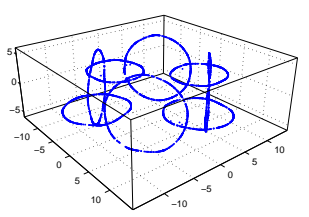

(b) The "chain of rings" dataset after denoising

Fig. 6. Denoising ability

\section{CONCLUSION}

Characterizing the statistical properties of the data is a first step in better understanding the problem at hand. In this paper, we have introduced a new cost function called the Information Theoretic Mean Shift algorithm that helps us "tune" to the principal curves and modes of the data; the two important features for manifold learning and clustering. Our algorithm is in essence a constrained entropy minimization problem. Since entropy takes all the moments of the data into account, we are in fact able to better characterize the statistical properties irrespective of the shape of the cluster. In this regard, our algorithm resembles the Information Bottleneck Method[11] but has a better intuitive reasoning.

GMS and GBMS algorithms were found to be special cases of our cost function. The GMS algorithm minimizes the "cross" entropy of the dataset $X$ and original dataset $X_{o}$. It was shown that this has a global solution corresponding to the modes of the pdf. The GBMS algorithm, on the other hand, arbitrarily reduces the entropy of the data making it suboptimal to find the modes. This information theoretical perspective of these two algorithms is indeed intuitively 


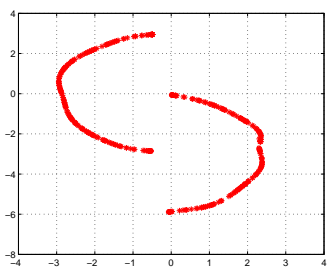

(a) $\lambda=2.8$

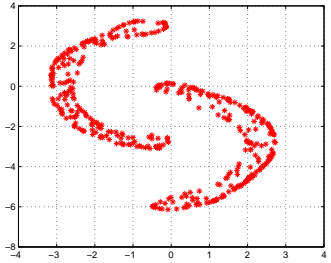

(d) $\lambda=9$

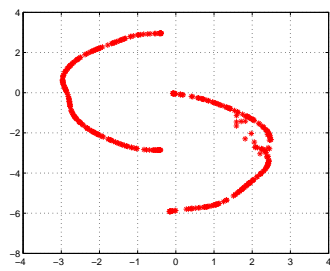

(b) $\lambda=3.5$

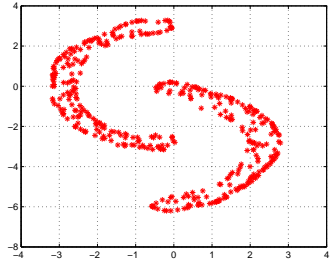

(e) $\lambda=13.1$

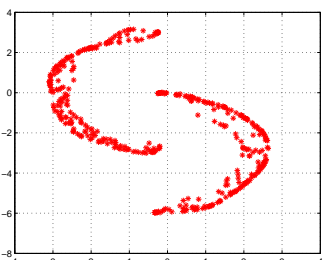

(c) $\lambda=5.5$

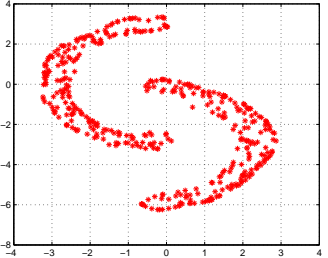

(f) $\lambda=20$

Fig. 5. Performance of our algorithm for increasing $\lambda$

appealing and attractive. Finally, we have shown that our algorithm is capable of finding the principal curve of the data making it extremely useful tool in manifold learning and denoising.

The challenge ahead is to find the optimal solution to the constrained optimization problem for a given CauchyScwartz distance. Another area of research is to figure out the reason of the instability of our fixed point algorithm for large values of $\lambda$. Finally we would also like to study the role of the kernel size $\sigma$ in tuning to a particular resolution for our analysis.

\section{ACKNOWLEDGEMENTS}

This work was partially supported by NSF grant ECS-0300340 and ECS-0601271.

\section{REFERENCES}

[1] K. Fukunaga and L.D. Hostetler, "The estimation of the gradient of a density function with applications in pattern recognition," IEEE Trans. on Information theory, vol. 21, no. 1, pp. 32-40, January 1975.

[2] Y. Cheng, "Mean shift, mode seeking and clustering," IEEE Trans. on Pattern analysis and Machine Intelligence, vol. 17, no. 8, pp. 790-799, August 1995.

[3] M.Á. Carreira-Perpiñán, "Mode-finding for mixtures of gaussian distributions," IEEE Trans. on Pattern analysis and Machine Intelligence, vol. 22, no. 11, pp. 1318-1323, November 2000.
[4] D. Comaniciu and P. Meer, "Mean shift: A robust approach toward feature space analysis," IEEE Trans. on Pattern analysis and Machine Intelligence, vol. 24, no. 5, pp. 603-619, May 2002.

[5] D. Comaniciu, V. Ramesh, and P. Meer, "Real-time tracking of non-rigid objects using mean shift," in Proceedings of IEEE Conf. Computer Vision and Pattern Recognition, June 2000, vol. 2, pp. 142-149.

[6] D. Comaniciu, Nonparametric Robust Methods for Computer Vision, Ph.D. thesis, Rutgers University, 1999.

[7] A. Renyi, "Some fundamental questions of information theory," in Selected Papers of Alfred Renyi, pp. 526-552. AKADEMIA KIADO, 1976.

[8] J. C. Principe, D. Xu, and J. Fisher, "Information theoretic learning," in Unsupervised Adaptive Filtering, Simon Haykin, Ed., pp. 265-319. JOHN WILEY, 2000.

[9] D. Erdogmus, Information Theoretic Learning: Renyi's Entropy and its Applications to Adaptive System Training, Ph.D. thesis, University of Florida, 2002.

[10] T. Hastie and W. Stuetzle, "Principal curves," Journal of the American Statistical Assiciation, vol. 84, no. 406, pp. 502-516, 1989.

[11] N. Tishby, F. Pereira, and W. Bialek, "The information bottleneck method," in Proceedings of the 37-th Annual Allerton Conference on Communication, Control and Computing, 1999, pp. 368-377. 